\title{
3D reconstruction identifies loci linked to variation in angle of individual sorghum leaves
}

Michael C. Tross ${ }^{\text {Corresp., } 1,2}$, Mathieu Gaillard ${ }^{3}$, Mackenzie Zwiener ${ }^{1}$, Chenyong Miao ${ }^{1}$, Ryleigh J. Grove ${ }^{1,4}$, Bosheng $\mathbf{L i}^{3}$, Bedrich Benes ${ }^{3,5}$, James C. Schnable ${ }^{\text {Corresp. 1, } 2}$

${ }^{1}$ Center for Plant Science Innovation and Department of Agronomy and Horticulture, University of Nebraska - Lincoln, Lincoln, Nebraska, United States

2 Complex Biosystems Graduate Program, University of Nebraska - Lincoln, Lincoln, Nebraska, United States

3 Computer Science, Purdue University, West Lafayette, Indiana, United States

4 Lincoln North Star High School, Lincoln, NE, United States

5 Department of Computer Graphics Technology, Purdue University, West Lafayette, Indiana, United States

Corresponding Authors: Michael C. Tross, James C. Schnable

Email address: mtross2@huskers.unl.edu, schnable@unl.edu

Selection for yield at high planting density has reshaped the leaf canopy of maize, improving photosynthetic productivity in high density settings. Further optimization of canopy architecture may be possible. However, measuring leaf angles, the widely studied component trait of leaf canopy architecture, by hand is a labor and time intensive process. Here, we use multiple, calibrated, 2D images to reconstruct the 3D geometry of individual sorghum plants using a voxel carving based algorithm. Automatic skeletonization and segmentation of these 3D geometries enable quantification of the angle of each leaf for each plant. The resulting measurements are both heritable and correlated with manually collected leaf angles. This automated and scaleable reconstruction approach was employed to measure leaf-by-leaf angles for a population of 366 sorghum plants at multiple time points, resulting in 971 successful reconstructions and 3,376 leaf angle measurements from individual leaves. A genome wide association study conducted using aggregated leaf angle data identified a known large effect leaf angle gene, several previously identified leaf angle QTL from a sorghum NAM population, and novel signals. Genome wide association studies conducted separately for three individual sorghum leaves identified a number of the same signals, a previously unreported signal shared across multiple leaves, and signals near the sorghum orthologs of two maize genes known to influence leaf angle. Automated measurement of individual leaves and mapping variants associated with leaf angle reduce the barriers to engineering ideal canopy architectures in sorghum and other grain crops. 


\title{
3D reconstruction identifies loci linked to variation in angle of individual sorghum leaves
}

\author{
Michael C. Tross ${ }^{1,2,+}$, Mathieu Gaillard ${ }^{3,+}$, Mackenzie Zweiner ${ }^{1}$, Chenyong Miao ${ }^{1, \dagger}$, \\ Ryleigh J. Grove ${ }^{1,5}$, Bosheng $\mathrm{Li}^{3}$, Bedrich Benes ${ }^{3,4}$, and James C. Schnable ${ }^{1,2^{*}}$ \\ ${ }^{1}$ Center for Plant Science Innovation and Department of Agronomy and Horticulture, University of Nebraska-Lincoln, \\ Lincoln, NE, USA \\ ${ }^{2}$ Complex Biosystems Graduate Program, University of Nebraska-Lincoln, Lincoln, NE, USA \\ ${ }^{3}$ Computer Science, Purdue University, West Lafayette, IN, USA \\ ${ }^{4}$ Department of Computer Graphics Technology, Purdue University, West Lafayette, IN, USA \\ ${ }^{5}$ Lincoln North Star High School, Lincoln, NE, USA \\ †resent Affiliation: Bayer CropScience AG \\ +Authors contributed equally \\ *schnable@unl.edu
}

\begin{abstract}
14 ABSTRACT
Selection for yield at high planting density has reshaped the leaf canopy of maize, improving photosynthetic productivity in high density settings. Further optimization of canopy architecture may be possible. However, measuring leaf angles, the widely studied component trait of leaf canopy architecture, by hand is a labor and time intensive process. Here, we use multiple, calibrated, 2D images to reconstruct the 3D geometry of individual sorghum plants using a voxel carving based algorithm. Automatic skeletonization and segmentation of these 3D geometries enable quantification of the angle of each leaf for each plant. The resulting measurements are both heritable and correlated with manually collected leaf angles. This automated and scaleable reconstruction approach was employed to measure leaf-by-leaf angles for a population of 366 sorghum plants at multiple time points, resulting in 971 successful reconstructions and 3,376 leaf angle measurements from individual leaves. A genome wide association study conducted using aggregated leaf angle data identified a known large effect leaf angle gene, several previously identified leaf angle QTL from a sorghum NAM population, and novel signals. Genome wide association studies conducted separately for three individual sorghum leaves identified a number of the same signals, a previously unreported signal shared across multiple leaves, and signals near the sorghum orthologs of two maize genes known to influence leaf angle. Automated measurement of individual leaves and mapping variants associated with leaf angle reduce the barriers to engineering ideal canopy architectures in sorghum and other grain crops.
\end{abstract}

\section{Introduction}

Efforts to increase yield in maize in the United States have hinged in large part, not on breeding individual plants which produce more grain, but breeding plants which can tolerate being grown more closely together, allowing farmers to grow more plants on the same amount of land [1,2]. Retrospective phenotypic and genetic analysis has shown that breeders inadvertently selected for maize varieties with more erect leaves as they selected for plants able to grow and produce grain at high densities [3]. When a corn plant produces leaves parallel to the ground, the photosynthetic apparatus is often overwhelmed in high light conditions, forcing it expend energy dissipating light energy, rather than capturing all of the energy via photosynthesis [4]. On an erect leaf, the same amount of incident light is distributed across more total surface area. This increases the amount of light a plant can effectively capture and translate into stored chemical energy via photosynthesis before being overwhelmed and dissipating the excess light energy [5]. In maize, the classical liguleless mutants produce plants with extremely erect leaves [6,7]. Introgressing a mutant allele of the liguleless 2 gene into 1960s era hybrids produced yield increases of $40 \%$ at high planting densities relative to near isogenic hybrids with the wild type allele of liguleless $2[8,9]$. Breeding for yield under high density has resulted in the leaf angle of modern maize hybrids being roughly $30^{\circ}$ more erect [10]. More erect leaf architecture allows for denser planting, thus increasing yield per acre of land $[3,11]$

Given its important contribution to advances in yield, the genetic control of leaf angle in grain crops has been extensively investigated through both classical and quantitative genetics in maize, as well as other grain crops. Natural allelic variation in both liguleless 1 and liguleless 2 contributes to variation in leaf angle among maize inbreds [12]. ZmTAC1 was first identified as a quantitative trait locus for leaf angle variation segregating in Chinese maize populations and encodes an ortholog of the

Peer) reviewing PDF | (2021:09:65925:1:0:NEW 10 Nov 2021) 1 
rice TAC1 gene. It is primarily expressed in the leaf sheath of maize and contributes to the leaf regulatory pathway, where it is believed to be affiliated with erect angles and a more compact architecture [13]. Liguleness-narrow (LGN-R) was recovered from an EMS mutant screeen and encodes a kinase upstream of liguleless 1 and liguleless 2 and mutant alleles produce extremely erect leaves [14]. ZmCLA4 was mapped through fine mapping of a QTL for leaf angle and encodes an ortholog of the lazyl gene from rice [15]. The paralogous mutants drooping leafl and drooping leaf2 encode YABBY transcription factors and were initially identified by classical genetics, however quantitative genetics analyses have linked these genes to natural variation in leaf angle in maize populations [16]. The upright plant architecture1 (UPA1) and upright plant architecture2 (UPA2) QTL identified in a cross between maize and its wild progentior were cloned by fine mapping, revealing that upright plant architecture 1 is explained by allelic variation in brassinosteroid $C-6$ oxidase 1 and upright plant architecture 2 results from allelic variation in ZmRAVL1 e [17]. ZmIBH1 and ZmILI1 are two basic helix-loop-helix transcription factors located on maize chromosome 2 where each appear to regulate natural variation in maize leaf angle [18,19]. As of 2017, a total of approximately 12 cloned maize mutants were associated with changes in leaf angle and 19 cloned mutants in rice were associated with changes in leaf angle [20]. In sorghum, a large effect mutation that also alters plant stature, $d w a r f 3$, has been shown to alter the angle of sorghum leaves by more than 30 degrees [21].

Despite the demonstrated importance of leaf angle for increasing yields under high planting densities across a range of grain crops, investigation of the control of leaf angle variation in sorghum has been comparatively limited. Prior to the sequencing of the sorghum genome, three QTL for leaf angle were identified in a population of 137 sorghum RILs, one of which corresponded to the $d$ warf3 gene [22]. A later QTL study employing more markers and a larger number of RILs was able to identify the effects of $d$ warf3 in the angle of two sequential leaves and among both field and greenhouse grown plants as well as smaller and less consistently detected QTL on chromosomes 1, 3, and 5 [21]. A study of leaf angle using manually collected leaf angle scores from 315 accessions of the sorghum association panel [23] identified a significant signal from the $d w a r f 3$ locus and several additional scattered signals at the threshold of statistical significance [24]. More recently, a study which employed manual scores of leaf erectness in a 2,200 line sorghum nested association panel identified a total of sixteen significant signals scattered across the genome [25]. Efforts have been made to automate the scoring of sorghum leaf angle. Leaf angle can be roughly estimated under field conditions by examining the overall width of rows of sorghum plants, a trait which can be measured from current robotic platforms [26]. When working with individual plants in a greenhouse setting, depth camera data collected from 12 viewing angles was sufficient to reconstruct sorghum plant meshes from a panel of 99 recombinant inbred lines, enabling the detection of the known effect of the $d$ warf3 gene on leaf angle via QTL mapping [27]. The push towards more automated approaches to measuring leaf angle in sorghum is the result of a significant bottleneck introduced by manual measurement which is labor intensive and can be error prone.

Current methods for automated measurements can track relative changes in leaf angle over time are unable to consistently measure leaf angles across different plants with differing phyllotaxy in the case of 2D measurements [28] - although it should be noted that this challenge was overcome in maize using sufficient numbers of 2D images taken from different angles [29] - or require additional dedicated instrumentation such as depth cameras [27]. Here we employ a recently published voxel carving based method for 3D reconstruction from conventional RGB photos [30] and a 3D skeletonization and leaf segmentation algorithm developed to work with the same voxel data [31]. We extend the use of these two algorithms to allow for the measurement of the angle of each leaf of a plant and employ data on the angles of multiple leaves across multiple time points from a sorghum association panel [23] to identify both known and previously unknown genetic loci controlling natural variation in leaf angle in sorghum.

\section{Methods}

\section{Image Acquisition and Annotation}

A set of 366 individual plants from 236 genotypes of the sorghum association panel [23] were grown and imaged using the University of Nebraska-Lincoln's automated phenotyping facility [32]. Growth conditions and details of the image acquisition were previously described in [30]. RGB images were taken on April 11th (47 days after planting), 13th (49 days after planting) and 16th (52 days after planting) of 2018, using a Basler pia2400-17gc camera with a c6z1218m3-5 Pentax TV zoom lens. These images were taken at five different side view angles: $0^{\circ}, 72^{\circ}, 144^{\circ}, 216^{\circ}$, and $288^{\circ}$ side views and with a single additional image taken from directly overhead. Each image had a resolution of 2,454 $\times 2,056$ pixels. The total number of side view images was dictated by the overall speed of the imaging system as the slowest imaging step dictated the time required to image all plants in the greenhouse. At the time, five side views was the maximum number which could be acquired by the RGB camera in the time required to capture one hyperspectral image which was the rate limiting step on data acquisition [32]. Given this constraint, the angles $0^{\circ}, 72^{\circ}, 144^{\circ}, 216^{\circ}$, and $288^{\circ}$ were selected to capture five equidistant perspectives around a full $360^{\circ}$ range of rotation. Images were calibrated as previously described [30]. Briefly, the calibration we applied corrects for the fact that pots are not perfectly centered on the axis of rotation of the turntable used to capture photos of the same plant from multiple angles and the axis of rotation for the turntable does not correspond to the optical center of the camera. 
Two sorghum lines representing extremely different leaf architectures were selected and grown for paired manual and automated phenotyping: Btx623 (erect leaf architecture) and AS 4601 Pawaga (non-erect leaf architecure). Manual measurements of leaf angles were taken with a protractor on January 13th of 2021. Plants were imaged at three time points January 12th (70 days after planting), 13th and 14th 2021. These images were employed for 3D reconstruction and automated leaf angle measurements. Between 2018 and 2021 the physical RGB camera and lens employed by the Nebraska automated phenotyping greenhouse was replaced increasing the resolution and changing the focal length.

\section{Plant Voxelization and 3D Skeletonization}

A 3D volumetric grid was reconstructed for each plant on each day using the five side view images from different angles and single top view image described above and an extended voxel carving algorithm [30]. The algorithm employed segmented images from each view to carve a voxel grid with a resolution of $512^{3}$ voxels. The voxels remaining after carving were used to generate a 3D skeleton of the plant [31].

Briefly, the generic voxel thinning algorithm implemented in the DGTal library was first run on the voxel set $\mathscr{V}$. This algorithm iteratively removes voxels from the reconstructed plant $\mathscr{V}$ until only its curve skeleton $\mathscr{S}_{\text {raw }}$ remains. This curve skeleton is a collection of curves whose shape faithfully represents the reconstructed plant. However, the curve skeleton $\mathscr{S}_{\text {raw }}$ includes some small spurious branches that do not correspond to separate organs of the plant being reconstructed. A machine learning classifier was run on the curve skeleton $\mathscr{S}_{\text {raw }}$ to distinguish between spurious branches and branches that correspond to real leaves with the spurious branches being discarded [31]. It is possible that the voxel grid contains more than one connected component i.e. the reconstructed plant is disconnected. In this case, the skeletonization algorithm will automatically connect branches together to form the plant skeleton. We denoted the final plant skeleton $\mathscr{S}$. Finally, the skeleton $\mathscr{S}$ is post-processed to identify the stem $\mathscr{T}$ and each individual leaf $\mathscr{L}=\left\{L_{1}, L_{2}, \ldots, L_{n}\right\}$. To differentiate voxels that are part of the stem or individual leaves, we consider that 1) any voxel that is shared by at least two branches is part of the stem, and 2) any voxel that is part of only one branch belong to the corresponding leaf [31]. Leaves were numbered based on the height of each leaf's leaf-stem junction with the first leave being the leaf which joins the stem at the lowest point and the last leaf being the detected leaf which joins the stem at the highest point.

\section{Leaf Angle Measurement from 3D Skeletons}

The angle of individual leaves is measured in a three step process. In the first step, the principal direction $\overrightarrow{v_{1, \mathscr{T}}}$ and the second direction $v_{2, \mathscr{T}}$ of the stem are identified and measured by analyzing the 3D coordinates of voxels assigned to the stem of the plant skeleton $\mathscr{T}$ using PCA (Principal Component Analysis). In the second step, the principal direction $\vec{L}_{n}$ of the first 20 voxels of each leaf (the 20 voxels after the leaf-stem junction) $L_{n}$ are computed, also through the use of the principal component analysis. In the final step, the principal directions of the stem and leaf are compared to compute two angles: 1) The polar angle $\theta \in\left[0^{\circ}, 180^{\circ}\right]$, which is the angle in the vertical plane, and 2) the azimutal angle $\phi \in\left[0^{\circ}, 360^{\circ}\right]$, which is the angle in the plane formed by $\overrightarrow{v_{1, \mathscr{T}}}$ and $\overrightarrow{v_{2, \mathscr{T}}}$. The angle $\theta$ is computed as the angle between the two 3-D vectors $\vec{v}_{L_{n}}$ and $v_{1, \mathscr{T}}$. The angle $\phi$ is computed as the angle between $v_{2, \mathscr{T}}$ and the projection of the leaf direction in the horizontal plane $\vec{v}_{L_{n}}-\left(\overrightarrow{v_{L_{n}}} \cdot v_{1, \mathscr{T}}\right) v_{1, \mathscr{T}}$.

\section{Leaf Angle Measurements From 2D Images}

For each plant at each time point, all captured viewing angles were segmented into plant and not-plant using the published PlantCV protocol [33,34]. Briefly, images were converted to grayscale, blurred, cropped to remove pots and backgrounds. These gray scale images were then converted to binary segmented images using the PlantCV thresholding function. Plant width was calculated as the distance between the left-most and right-most plant pixels in each image, and for each plant on each day the viewing angle with the greatest plant width was selected as the one most likely to represent a plane of phyllotaxy roughly perpendicular to the viewing angle of the camera. This widest image was skeletonized, the skeleton divided into leaf segments and the insertion angles of each leaf segment were calculated following the "morphology" protocol workflow from PlantCV's documentation [34].

\section{Heritability and Genome-wide Association}

Broad sense heritability was calculated using 48 replicated genotypes included in the study using the equation $H^{2}=\sigma_{G}^{2} /\left(\sigma_{G}^{2}+\right.$ $\left.\sigma_{e}^{2} / 2\right)$, where $\sigma_{G}^{2}$ is the total amount of variance that is due to genetics and $\sigma_{e}^{2}$ is the total residual variance. The variance components were extracted from the linear model: $y_{i}=\mu+t_{i}+e_{i}$, where $y_{i}$ is the mean yield of the genotype, $\mu$ is the overall mean, $t_{i}$ is the effect of genotype $\mathrm{i}$ and $e_{i}$ is the residual error of genotype $\mathrm{i}$. This was implemented in the software package lme4 (v1.1-23) [35].

Best linear unbiased predictors (BLUPs) [36] were calculated for each set of leaf angle measurements used for GWAS with leaf angle treated as a random effect using the same linear model: $y_{i}=\mu+t_{i}+e_{i}$. Any genotype with a BLUP more than 5 standard deviations away from the mean was treated as an outlier and removed from downstream analysis. Each outlier value was checked against image data to confirm the extreme values resulted from the plant itself and not reconstruction errors. In 

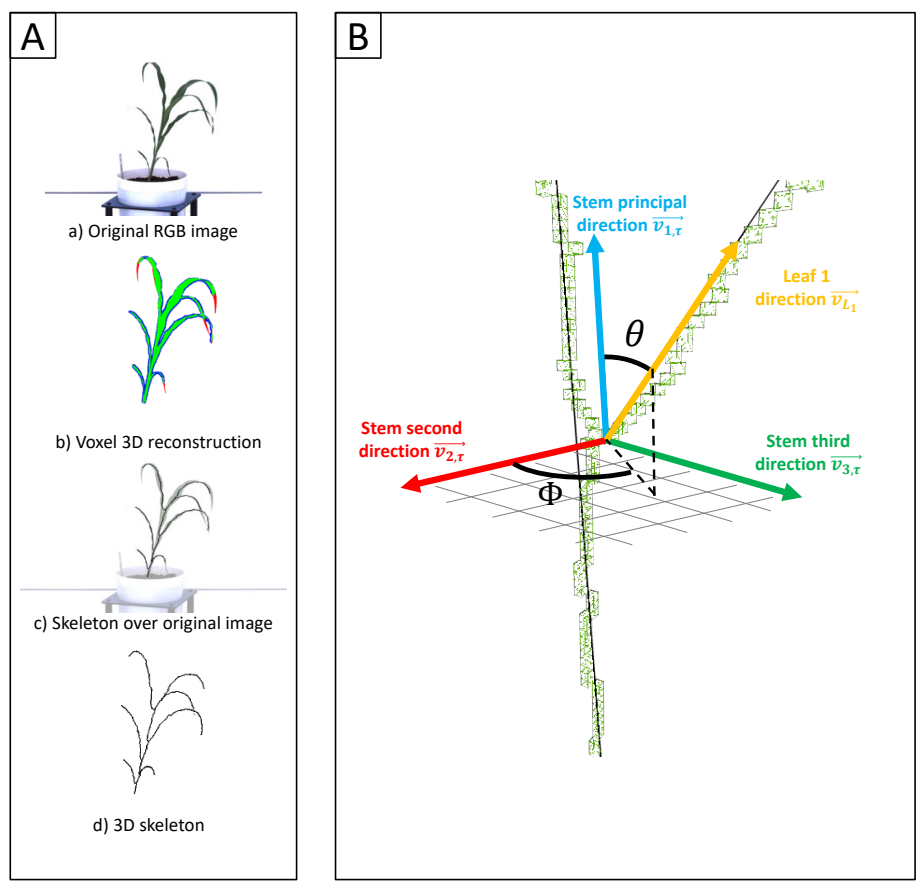

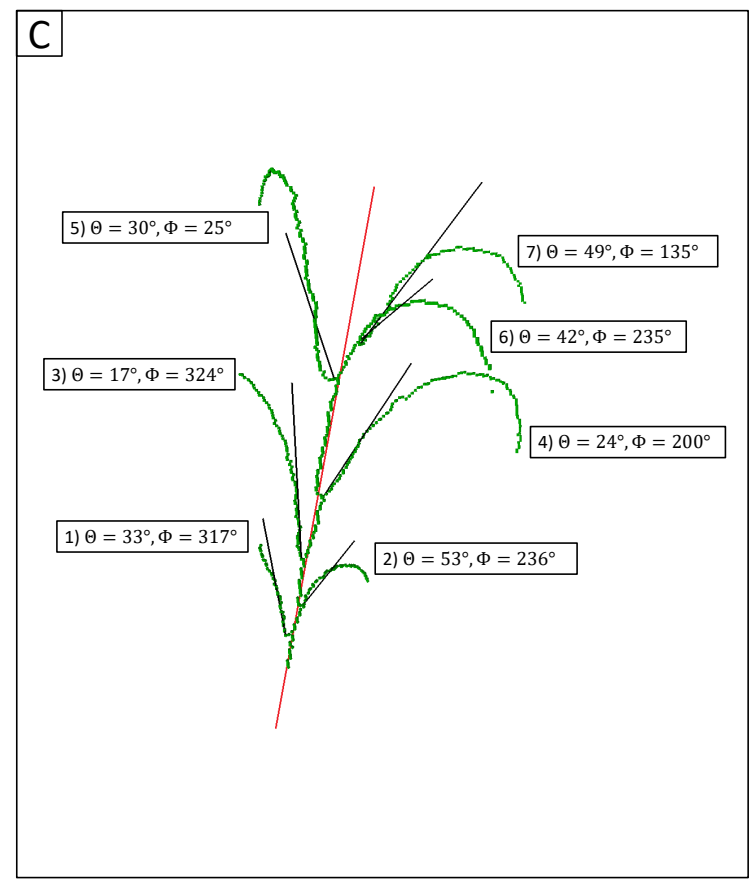

Figure 1. Measurement of individual leaf angles from 3D reconstructions of sorghum plants. A) a) An example of one of the six view 2D images - five side views and one top view - used to reconstruct the 3D volume of the plant using voxel carving. b) Evaluation of the quality of the voxel reconstruction by comparison the initial segmentation of this 2D image for plant and not-plant pixels to a reprojection of the voxel reconstruction as a 2D image viewed from the same perspective. Green pixels mark overlap between these two images. Red pixels are places identified as part of the plant in the original segmented image but not the reprojection. Blue pixels are places which are part of the projection but were not identified as part of the plant in the original 2D segmentation. c) A 3D skeleton (black lines) fit to the voxel reconstruction. d) Overlay of the 3D skeleton on the original RGB image from panel a. B) Measurement of individual sorghum leaf angles in 3D space. Separate vectors are generated for the stem (blue) and leaf (yellow) using the voxel-based skeletons of each organ. Leaf angle was defined as the polar angle $\theta$ (the angle with regard to the stem principal direction). These measurements also reconstructed a second azimutal angle $\phi$ (angle in the plane formed by $\overrightarrow{v_{1, \mathscr{T}}}$ and $v_{2, \mathscr{T}}$ ). C) The plant skeleton with measured angles for each leaf indicated.The solid red line indicates the stem principal direction while black lines mark the principal directions for each leaf. 


\section{Measurements from 3D reconstructions of sorghum plants}

Initially two lines representing phenotypic extremes for leaf angle in the sorghum association population were selected to evaluate leaf angle measurement: BTx623, the reference genotype for sorghum which produces erect leaves ( Figure S1A), and AS 4601 Pawaga which produces non-erect leaves ( Figure S1B). Six plants were grown to late vegetative stage, the angles of the first four non-senesced leaves were manually measured, and the plants were imaged using a previously described automated greenhouse imaging system [32]. Sets of 2D images collected from each plant from multiple viewing angles were processed to generate 3D voxel reconstructions using the method described in [31] (Figure 1A). Three dimensional skeletonization and pruning was employed to identify individual leaves [31] (Figure 1B). And the angle of individual leaves relative to the principle direction of the stem was quantified from these segmented skeletonized leaves (Figure 1C). The manual and Automated 3D measurements of median leaf angles across the first four leaves were highly positively correlated $r=0.98$ with manual measurements taken with a digital caliper (Figure S3A; S2). Manual and automated measurements for individual leaves exhibited a modestly lower but still high correlation $r=0.86$ (Figure S3B).

Having evaluated the performance of this 3D approach to leaf angle measurement on a small set of plants with ground truth manual measurement, the algorithm was next applied to a much larger experiment from which ground truth measurements had not been collected: An image dataset collected at multiple time points from a total of 366 sorghum plants representing 236 unique genotypes were used to create 1092 3D reconstructions, each representing a single plant on a single day. A total of 119 $3 \mathrm{D}$ reconstructions failed. In 39 cases corresponding to 13 pots on each of the three time points no plant was present in any image, presumably the result of failed germination or the inclusion of calibration pots. In 35 cases calibration was unsuccessful. In the remaining 45 cases either the cocordance between the original segmented 2D images and simulated 2D segmented images generated from 3D reconstructions was below 0.8 [30], or the 3D skeletonization algorithm was not able to confidently identify the soil-stem junction [31]. Of the remaining 973 3D reconstructions, 2 were removed based on unexpectedly extreme leaf angle values. Manual evaluation of these two outlier reconstructions suggested that one resulted from a small plant with a large tiller (Figure S4A). The reason for the poor reconstruction of the second outlier plant was not immediately apparent (Figure S4B).

Sorghum plant reconstructions for the remaining 971 unique plant/timepoint combinations contained between 3 and 19 predicted leaves, with a median of 10 (Figure S5). Median leaf angle declined, e.g. became more erect, starting from the bottom most leaf and proceeding upwards to leaf six (Figure S6). Leaf angles measured from 3D skeletons for leaves above leaf six exhibited a much wider distribution of values, which may reflect the difficulty of accurately quantifying leaf angle for leaves still emerging from the leaf whorl where the ligule/auricle junction is not yet visible. It was not possible to automate the separation of mature and emerging leaves, so it was unclear how much accuracy was lost for uppermost leaves.

As this analysis was conducted using a previously collected sorghum image dataset for which ground truth leaf angles were not manually scored, it was not possible to directly evaluate how well this larger set of measured leaf angles represented the angles which would have been measured by hand from the exact same plants. However, because the genotypes used in this study were largely homozygous inbred lines, it was possible to grow plants with identical or near identical genomes to the same stage of development and collect ground truth measurements from these plants. Independent grow outs of the same set of genotypes do not produce perfectly equivalent phenotypes. Measurements of plant leaf angle collected in Iowa and Nebraska from the same population were compared in order to estimate how much correlation in leaf angle would be expected between two independently grown sets of plants at the same stage of development - reproductive stage in this case - collected in different environments. The correlation between these two manual datasets data was significant $(r=0.60 ; p=0.0002$; pearson). Leaf angle measurement were manually scored from a set of 79 lines from the sorghum association panel grown to a similar late vegetative stage of development under greenhouse conditions were significantly correlated with automated 3D measurements $(\mathrm{r}=0.53 ; \mathrm{p}=0.0016$; pearson) (Figure S7). However, analysis of the same image data using conventional 2D approaches to quantifying leaf angle from single images produced values which were not significantly correlated with either manual measures of the same genotypes at the same stage, or leaf angles measured from 3D reconstructions using the same image data (Figure S7). Manual measurements of plant leaf angle between Iowa and Nebraska datasets were anticipated to be higher than the automatic 3D versus manual measurements of the greenhouse plants where different plants of the same genotypes were compared. The leaf angles for each genotype in both the Iowa datasets and Nebraska dataset of mature plants were the means of up to 18 and 2 replicates of genotypes respectively. This would reduce environmental, genotype by environment and many other residual effects. The dataset composing of $3 \mathrm{D}$ reconstructed plants and that of the 79 lines under greenhouse conditions generally represented measurements of multiple leaves from only a single plant.

Assuming random error in quantification, as opposed to genetically controlled error [42], if the angles of upper leaves are measured with lower accuracy, the heritability - i.e. the proportion of total variance attributable to differences between genotypes - of leaf angle measurements for these leaves would likely be lower. A total of 48 sorghum genotypes were replicated between two and eight times each among the 366 unique sorghum plants imaged. Only the first seven leaves of each plant were considered in our analysis. Higher leaves were generally not mature as evident by the auricle not being extended from the 
sheath. This would limit the accuracy of leaf angle measurements of those after the seventh leaf. The heritability of 3D leaf angle measurements tended to be higher for the first five leaves than for leaves six or seven on each of the three time points measured (Figure S8A). The median measurement for the angle of a particular leaf across three time points tended to be more heritable than measurements of the same leaf at individual time points, consistent with expectations for repeated measurements with independent error (Figure S8A). Aggregating measurements across sequential leaves also tended to increase heritability relative to single leaf measurements (Figure S8B). Of the aggregated leaf ranges within the aggregated time points, the median of leaves 1 to 4 had the highest heritability value of 0.72 (Figure S8B). The heritability of measurements of leaf angle obtained from conventional 2D analysis of the same set of sorghum images were generally much lower than 3D measurements with no genetic effect detected in a number of cases (FigureS9).

A)

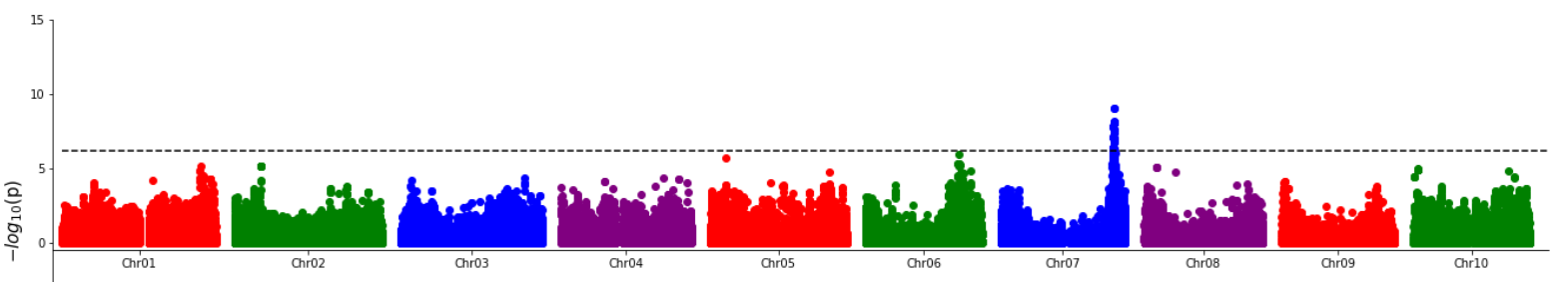

B)

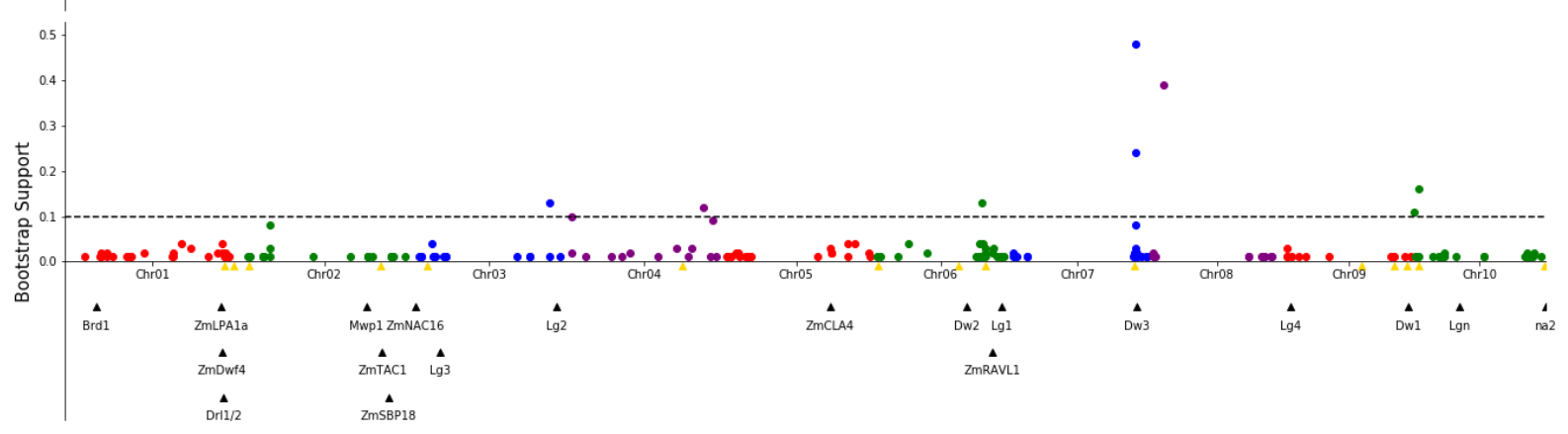

Figure 2. Genetic markers significantly associated with variation in sorghum leaf angle aggregiated across leaves and time points. A) Results of a genome wide association study conducted using the GEMMA algorithm. Each point indicates the physical position and statistical significance of an individual marker. Dashed black line indicates a genome wide threshold for statistical significance of $6.39 \times 10^{-7}$ resulting from a bonferroni correction using an effective SNP number of 78,251 (See Methods). B) Results of a resampling based assessment of associations identified using the FarmCPU GWAS algorithm. Each circle represents an individual genetic marker which was statistically significantly associated with leaf angle variation in at least one of 100 FarmCPU analysis conducted using subsets of the total phenotypic dataset. $x$-axis position indicates the physical position of the marker and y-axis position indicates the proportion of the 100 analysis in which the marker was identified as statistically significant. Dashed black line indicates a threshold cutoff for stable and significant associations which were detected in $>10 \%$ of total resampling analyses. Yellow triangles indicate the locations of significant QTL for leaf erectness detected in an analysis of a sorghum NAM population [25]. Black triangles indicate locations of a set of cloned sorghum genes or the locations of the syntenic ortholog in sorghum of maize or rice genes known to influence variation in leaf angle in those species.

\section{Genetic loci associated with leaf angle variation in the SAP}

A published set of SNP markers for the sorghum association panel [37] was employed to conduct a genome wide association study for both the 3D and 2D aggregated leaf angle data across the first four leaves and all three time points. Analysis with GEMMA [39], a mixed linear model based algorithm, did not identify any significant markers for the automated 2D angles (Figure S10) but for 3D derived angles, identified a single large peak for leaf angle located on chromosome 7 between 59.8 MB and 59.9 MB. This position corresponds to the genomic location of $d w a r f 3$ (Sobic.007G163800), an ATP-binding cassette (ABC) transporter involved in auxin export [43] that has been previously associated with variation in leaf angle in sorghum RIL populations [21] and an association study [26]. As $d w a r f 3$ is a large effect locus with functionally variable alleles segregating at high frequencies in this population, we speculated that the effects of additional loci on angle might be masked in the 
MLM-based GWAS results (Figure2A). The FarmCPU algorithm, a multi-locus GWAS algorithm that can control for large effect loci to increase the likelihood of identifying additional true positive signals, was employed to analyze the same trait and genotype datasets. The significance of FarmCPU results were controlled for using a resampling-based strategy [44]. A total of eight markers clustered at six locations in the genome showed significant associations ( resample model inclusion probability $($ RMIP) $>0.1$ ) with median angle of leaves $1-4$ across all three time points in $>10 \%$ of resampled FarmCPU GWAS results, including the $d$ warf3 locus (Figure2B). None of the five non- $d$ warf3 clusters were associated with a set of 19 a priori candidate sorghum genes identified based on syntenic orthology to genes known to influence leaf angle in maize or rice. However, three of the six clusters associated with variation in leaf angle were consistent with QTL detected in a nested association mapping study for leaf erectness using a population of 2,200 sorghum RILs [25].

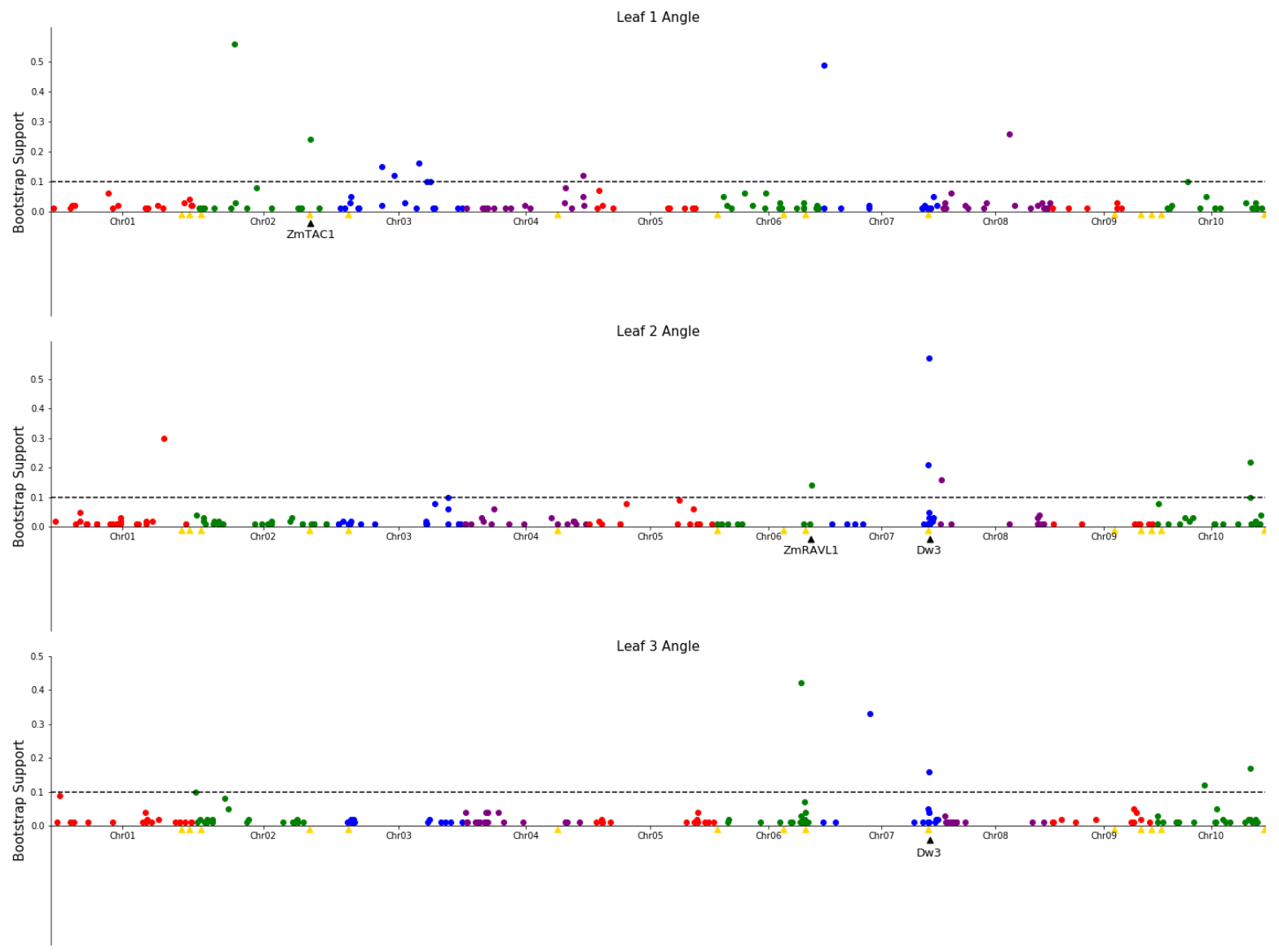

Figure 3. Genetic markers significantly associated with variation in the angle of leaves $\mathbf{1 ,}$, or 3 . A resampling based assessment of associations identified using the FarmCPU GWAS algorithm using the median leaf angle for leaf 1, 2, or 3 across all three time points. As in Figure 2, the dashed black line indicates a threshold cutoff for stable and significant associations which were detected in $>10 \%$ of total resampling analyses. Yellow triangles indicate the locations of significant QTL for leaf erectness detected in an analysis of a sorghum NAM population [25]. Black triangles indicate locations of a set of cloned sorghum genes or the locations of the syntenic ortholog in sorghum of maize genes which are both known to influence variation in leaf angle in maize and are located near significantly trait associated SNPs in this study.

The reconstruction of full architectures of individual plants provided the opportunity to assess how much overlap was present in the loci showing significant associations with variation in leaf angle for individual leaves. Separate analyses were conducted for the angle of the first, second, third, and fourth identified leaves of each sorghum plant. It must be noted that these likely do not correspond to the leaves which would be called leaf 1, leaf 2, leaf 3, and 4 as early sorghum seedling leaves senesce and die as the plant continues to develop. However, the first identified leaf should always be an earlier developing leaf than the second and third and and so on. Eight total markers showed significant associations in $>10 \%$ of sub-sampled GWAS results for the angle of the first identified leaf although three of these were grouped together in the pericentromeric region of chromosome 3 and may represent a single underlying singla, five clusters of $\geq 1$ SNPs showed significant associations in $>10 \%$ of sub-sampled GWAS results for the second identified leaf, five showed significant associations in $>10 \%$ of sub-sampled 
GWAS results for the third identified leaf, and no significant signals were identified for the fourth identified leaf. The signal associated with the $d w a r f 3$ locus was detectable for the second and third leaves but not the first leaf. One of the six significant clusters identified in GWAS for the angle of the first leaf was located on sorghum chromosome 2, 420 kilobases from the sorghum ortholog (Sobic.002G272400) of ZmTac1 [13]. One of the five significant associations for the second leaf was located at 54.99MB, 503 kilobases from the sorghum ortholog (Sobic.006G190400) of the maize gene UPA2/ZmRAVL1 [17]. In addition to the $d w a r f 3$ locus, a second shared signal was identified between GWAS for the angle of the second identified leaf and GWAS for the angle of the third identified leaf at position $52.75 \mathrm{MB}$ on sorghum chromosome 10 . This hit did not correspond to either published loci associated with leaf erectness in the sorghum NAM population or sorghum orthologs of candidate leaf angle regulatory genes from maize or rice. Mixed linear model based, leaf by leaf GWAS results, showed 3 significant associations for leaf 2 and one for leaf 3 (Figure S11). Two of the associations for leaf 2, one on chromosome 7 and the other on 10 was in close proximity to signifcant SNPs via the leaf by leaf FarmCPU resampling (5.031 and 29.997 kilobase differences respectively).The association for leaf 3 , on chromosome 6 , was also in close proximity to a significant SNP found in the FarmCPU resampling method (34.572 kilobase difference).

\section{Discussion}

Leaf angle has been shown to play a role in determining photosynthetic productivity of grain crops at differing planting densities. In sorghum, quantitative genetic investigation of the genes controlling natural variation in leaf angle has been limited by the labor intensive nature of manual leaf angle measurements. Here we employed voxel-based 3D reconstruction of greenhouse grown plants combined with 3D skeletonization to quantify leaf angles for each individual leaf of 366 sorghum plants at three time points, representing 971 individual 3D reconstructions - after removing failed reconstructions - and 3,376 total individual leaves. We chose to focus on the angle between leaves and stems which more directly corresponds to manual measurements used by plant geneticists to identify genes controlling variation in leaf angle [20] (Figure S2). Automated measurements of the angle of lower leaves from 3D reconstructions - which tend to be fully expanded and ligulated - were positively correlated with manual measurements of the same plants in a small study (Figure S3), correlated with manual measurements of plants of the same genotype in a larger study (Figure S7), and heritable in a study employing the sorghum association population (Figure S8A).

Aggregated leaf angle data extracted from 3D reconstructions was sufficient to identify $d w a r f 3$ (Figure 2A), a locus with known effects on leaf angle in sorghum [21,27]. This known leaf angle gene could not be identified using leaf angle measured obtained from applying conventional 2D approaches to measuring leaf angles to the same image dataset. Analysis of the same dataset with the FarmCPU GWAS algorithm identified five additional signals, two of which were consistent with previously reported leaf angle QTL [25]. However, while the majority of previous quantitative genetic analyses of leaf angle in sorghum focused on either a single leaf or an average across leaves [20], our 3D reconstructions made it possible to conduct separate analyses for variants influencing the angle of the four lowest non-senesced leaves. Significant signals were identified for three of these four leaves including a known leaf angle variant from sorghum, signals near the sorghum orthologs of maize genes known to influence leaf angle, and a single independently identified for the angle of leaves two and three which was not linked to previous QTL in sorghum nor near orthologs of known leaf angle related genes in relatives. However, many loci showing significant associations with the angle of only one of the leaves tested.

It would be tempting to interpret this as evidence of largely independent genetic architectures controlling the angle of each individual sorghum leaf. More pessimistically the same result could be interpreted as many of the identified associations representing false positive associations. There is indeed evidence that genetic variants associated with upper and mid-level leaf angle in sorghum are only partially overlapping [26]. However, in this case we examined the angle of three sequential leaves rather than distinct parts of the sorghum canopy. In addition, as a result of different rates of senescence for juvenile leaves, there is no guarantee that the "leaf one" of two distinct sorghum genotypes in this study was a biologically equivalent leaf. Should we then conclude that the low degree of overlap between the trait associated SNPs for the angle leaves one, two and three results from a high rate of false positives? Not necessarily. For traits controlled by complex genetic architecture, such as leaf angle in sorghum, statistical approaches to GWAS must accept a high degree of false negatives in order to minimize false positives. In simulation studies using the sorghum association panel, for traits with the observed heritability of individual sorghum leaves controlled by as few as 64 total genetic variants, less than $40 \%$ of total variants are predicted to be discovered the FarmCPU algorithm - and even less using more conventional MLM based approaches [45]. The simplest interpretation of the low amount of overlap between the significant signals identified for the angle leaves 1,2, and 3 may that each represents a different subset of the true variant associations with lower leaf angle in sorghum, identified from independent datasets which all reflect a mixture of consistent genotype effects, genes with increasing or decreasing effects from lower to upper leaves, leaf by leaf non-genetic variation, and measurement error.

This study demonstrated that aggregating measurements of the same plant across multiple time points, even time points separated by only several days, can increase the proportion of variance explained by inter-genotype variation. By imaging 
in a controlled environment with artificial lighting, we were able to maintain largely constant lighting conditions across time points. Changes in illumination in field or greenhouse environments would make obtaining accurate segmentation across time points more challenging [46]. However, assuming accurate segmentation of 2D images, changing in lighting conditions would not alter the accuracy of 3D reconstruction based on the voxel carving approach employed here. To more accurately leverage data from multiple time points as well as reliably track changes in leaf angle over time in response to environmental changes, it would be necessary to track each leaf across successive reconstructions of the same individual plant. This is a computationally challenging for at least three reasons. Firstly, successive reconstructions of the same plant may not be aligned as a result of affine transformations resulting from rotation of the plant and pot. Secondly, leaves themselves grow and change over time. Individual leaves emerge from the whorl, lengthen and lower their observed angle with respect to the stem until the leaf reaches maturity. Later the lowest leaves will tend to senesce, wither, and die to the point they will no longer be detectable on subsequent 3D reconstructions. Thirdly, discrete events can occur which significantly alter the architecture of the plant, particularly the emergence of a newly detectable leaf from the whorl, or the senescence, withering, and death of lower leaves to the point they will no longer be detectable on subsequent 3D reconstructions.

There are conceptually straightforward computational approaches which could be adopted to address the first two challenges. Considering two successive reconstructions $P_{t}$ and $P_{t+1}$ of the same plant $P$ at time points $t$ and $t+1$, it is possible to find the optimal homography to align $P_{t}$ and $P_{t+1}$, minimizing the distance between them. Subsequently, it is possible to measure the distances between all pairs of leaves from $P_{t}$ to $P_{t+1}$, and compute a minimum matching between them (for example by using the Hungarian algorithm [47,48]). This would give us for each leaf of $P_{t}$, the corresponding leaf in $P_{t+1}$, while minimizing the total distance between all pairs of matched leaves. By successively tracking leaves from $P_{1}$ to $P_{2}$, then $P_{2}$ to $P_{3}$, all the way up to $P_{n}$, we track each leaf over the entire life span of the plant. Unfortunately, this method has one major drawback, it assumes the number and identity of leaves is constant between data collected from the same plant at different time points, causing it to fail when confronted with the third challenge outlined above: the emergence of new leaves or the senescence and death of old ones. Further work is needed to extend leaf tracking across multiple time points to take into account these discrete events which change the number and/or identity of detectable leaves present.

In conclusion, here we demonstrated the ability to quantify individual leaf level angle measurements in sorghum using 3D reconstructions. The method does not require specialized equipment such as depth cameras or LIDAR sensors and can scale to populations of hundreds of plants. We demonstrate that leaf angle measurements collected in this fashion can be used to identify both known and novel loci in the sorghum genome influencing overall leaf angle or the angle of individual sorghum leaves. 3D reconstruction and leaf angle measurement - as well as the measurement of other leaf properties including length, width, and curvature - across multiple stages of development can enable a more comprehensive understanding of the genetic determinants of sorghum canopy architecture and aid breeding or engineering of more productive and resource use efficient "smart canopies" [26].

\section{Acknowledgements}

Research reported in the publication was supported by the Foundation for Food and Agriculture Research (award number-Grant ID: 602757) to BB and JCS. The content of this publication is solely the responsibility of the authors and does not necessarily represent the official views of the FFAR. This work is supported by 2020-68013-32371 from the USDA National Institute of Food and Agriculture and by the Office of Science (BER), U.S. Department of Energy, Grant no. DE-SC0020355 to JCS.

\section{Author Contribution Statement}

BB and JCS conceived the project. MZ and CM generated the data MCT, MG, RJG, and BL conducted the analyses MCT, MG, $\mathrm{BB}$, and JCS wrote the paper.

\section{Data and Code availability}

The code for reconstruction and skeletonization is hosted on GitHub: https://github.com/cropsinsilico/ SorghumVoxelCarving. Raw images analyzed in this study have been deposited on Zenodo: http://doi.org/ 10.5281/zenodo.4426620. Phenotypic data, GWAS result files and code for main figures are located on GitHub: https://github.com/mtross2/Sorghum-3D-Reconstruction

\section{Conflicts of Interest}

J.C.S. has an equity interests in Data2Bio, a company that provides genotyping services using the same protocol employed for genotyping in this study. The authors affirm that they have no other conflicts of interest. 


\section{References}

1. Duvick, D. N. Genetic contributions to yield gains of us hybrid maize, 1930 to 1980 . Genet. contributions to yield gains five major crop plants 7, 15-47 (1984).

2. Tollenaar, M., Lee, E. et al. 2 strategies for enhancing grain yield in maize. Plant breeding reviews 34, 37-82 (2011).

3. Duvick, D. Genetic progress in yield of united states maize (zea mays 1.). Maydica 50, 193 (2005).

4. Murchie, E. H. \& Niyogi, K. K. Manipulation of photoprotection to improve plant photosynthesis. Plant physiology 155, 86-92 (2011).

5. Long, S. P., ZHU, X.-G., Naidu, S. L. \& Ort, D. R. Can improvement in photosynthesis increase crop yields? Plant, cell \& environment 29, 315-330 (2006).

6. Moreno, M. A., Harper, L. C., Krueger, R. W., Dellaporta, S. L. \& Freeling, M. liguleless1 encodes a nuclear-localized protein required for induction of ligules and auricles during maize leaf organogenesis. Genes \& development 11, 616-628 (1997).

7. Walsh, J., Waters, C. A. \& Freeling, M. The maize geneliguleless 2 encodes a basic leucine zipper protein involved in the establishment of the leaf blade-sheath boundary. Genes \& development 12, 208-218 (1998).

8. Pendleton, J., Smith, G., Winter, S. \& Johnston, T. Field investigations of the relationships of leaf angle in corn (zea mays 1.) to grain yield and apparent photosynthesis 1. Agron. J. 60, 422-424 (1968).

9. Lambert, R. \& Johnson, R. Leaf angle, tassel morphology, and the performance of maize hybrids 1. Crop. Sci. 18, 499-502 (1978).

10. Lauer, S. et al. Morphological changes in parental lines of pioneer brand maize hybrids in the us central corn belt. Crop. Sci. 52, 1033-1043 (2012).

11. Ma, D. et al. Changes in the morphological traits of maize genotypes in china between the 1950s and 2000s. Eur. journal agronomy 58, 1-10 (2014).

12. Tian, F. et al. Genome-wide association study of leaf architecture in the maize nested association mapping population. Nat. genetics 43, 159-162 (2011).

13. $\mathrm{Ku}$, L. et al. Cloning and characterization of a putative tac1 ortholog associated with leaf angle in maize (zea mays 1.). PLoS One 6, e20621 (2011).

14. Moon, J., Candela, H. \& Hake, S. The liguleless narrow mutation affects proximal-distal signaling and leaf growth. Development 140, 405-412 (2013).

15. Zhang, J. et al. The zmcla4 gene in the qla4-1 qtl controls leaf angle in maize (zea mays 1.). J. experimental botany 65, 5063-5076 (2014).

16. Strable, J. et al. Maize yabby genes drooping leaf 1 and drooping leaf 2 regulate plant architecture. The Plant Cell 29, 1622-1641 (2017).

17. Tian, J. et al. Teosinte ligule allele narrows plant architecture and enhances high-density maize yields. Science 365, 658-664 (2019).

18. Cao, Y. et al. Zmibh1-1 regulates plant architecture in maize. J. Exp. Bot. 71, 2943-2955 (2020).

19. Ren, Z. et al. Zmili1 regulates leaf angle by directly affecting liguleless1 expression in maize. Plant Biotechnol. J. 18, 881-883 (2020).

20. Mantilla-Perez, M. B. \& Salas Fernandez, M. G. Differential manipulation of leaf angle throughout the canopy: current status and prospects. J. Exp. Bot. 68, 5699-5717 (2017).

21. Truong, S. K., McCormick, R. F., Rooney, W. L. \& Mullet, J. E. Harnessing genetic variation in leaf angle to increase productivity of sorghum bicolor. Genetics 201, 1229-1238 (2015).

22. Hart, G., Schertz, K., Peng, Y. \& Syed, N. Genetic mapping of sorghum bicolor (1.) moench qtls that control variation in tillering and other morphological characters. Theor. Appl. Genet. 103, 1232-1242 (2001).

23. Casa, A. M. et al. Community resources and strategies for association mapping in sorghum. Crop. science 48, 30-40 (2008).

24. Zhao, J., Mantilla Perez, M. B., Hu, J. \& Salas Fernandez, M. G. Genome-wide association study for nine plant architecture traits in sorghum. The Plant Genome 9, 1-14 (2016). 
25. Olatoye, M. O., Hu, Z. \& Morris, G. P. Genome-wide mapping and prediction of plant architecture in a sorghum nested association mapping population. bioRxiv (2020).

26. Mantilla-Perez, M. B., Bao, Y., Tang, L., Schnable, P. S. \& Salas-Fernandez, M. G. Toward "smart canopy" sorghum: Discovery of the genetic control of leaf angle across layers. Plant Physiol. 184, 1927-1940 (2020).

27. McCormick, R. F., Truong, S. K. \& Mullet, J. E. 3d sorghum reconstructions from depth images identify qtl regulating shoot architecture. Plant physiology 172, 823-834 (2016).

28. Kenchanmane Raju, S. K. et al. Leaf angle extractor: A high-throughput image processing framework for leaf angle measurements in maize and sorghum. Appl. plant sciences 8, e11385 (2020).

29. Zhang, X. et al. High-throughput phenotyping and qtl mapping reveals the genetic architecture of maize plant growth. Plant physiology 173, 1554-1564 (2017).

30. Gaillard, M., Miao, C., Schnable, J. C. \& Benes, B. Voxel carving-based 3d reconstruction of sorghum identifies genetic determinants of light interception efficiency. Plant direct 4, e00255 (2020).

31. Gaillard, M., Miao, C., Schnable, J. \& Benes, B. Sorghum segmentation by skeleton extraction. In Computer VisionECCV 2020 Workshops, DOI: https://doi.org/10.1007/978-3-030-65414-6_21 (2020).

32. Ge, Y., Bai, G., Stoerger, V. \& Schnable, J. C. Temporal dynamics of maize plant growth, water use, and leaf water content using automated high throughput rgb and hyperspectral imaging. Comput. Electron. Agric. 127, 625-632 (2016).

33. Fahlgren, N. et al. A versatile phenotyping system and analytics platform reveals diverse temporal responses to water availability in setaria. Mol. plant 8, 1520-1535 (2015).

34. Gehan, M. A. et al. Plantev v2: Image analysis software for high-throughput plant phenotyping. PeerJ 5, e4088 (2017).

35. Bates, D., Mächler, M., Bolker, B. \& Walker, S. Fitting linear mixed-effects models using lme4. J. Stat. Softw. 67, 1-48, DOI: 10.18637/jss.v067.i01 (2015).

36. Robinson, G. K. et al. That blup is a good thing: the estimation of random effects. Stat. science 6, 15-32 (1991).

37. Miao, C., Xu, Y., Liu, S., Schnable, P. S. \& Schnable, J. C. Increased power and accuracy of causal locus identification in time series genome-wide association in sorghum. Plant Physiol. 183, 1898-1909 (2020).

38. Yu, J., Pressoir, G. \& Briggs, W. Vroh bi i, yamasaki m, doebley jf, mcmullen md, gaut bs, nielsen dm, holland jb, et al. a unified mixed-model method for association mapping that accounts for multiple levels of relatedness. Nat Genet. 38, 203-208 (2006).

39. Zhou, X. \& Stephens, M. Genome-wide efficient mixed-model analysis for association studies. Nat. genetics $\mathbf{4 4 ,} 821$ (2012).

40. Yin, L. et al. rmvp: A memory-efficient, visualization-enhanced, and parallel-accelerated tool for genome-wide association study. bioRxiv (2020).

41. Li, M.-X., Yeung, J. M., Cherny, S. S. \& Sham, P. C. Evaluating the effective numbers of independent tests and significant p-value thresholds in commercial genotyping arrays and public imputation reference datasets. Hum. genetics 131, 747-756 (2012).

42. Liang, Z. et al. Conventional and hyperspectral time-series imaging of maize lines widely used in field trials. GigaScience 7, gix117 (2017).

43. Multani, D. S. et al. Loss of an mdr transporter in compact stalks of maize br2 and sorghum dw3 mutants. Science 302, 81-84 (2003).

44. Valdar, W., Holmes, C. C., Mott, R. \& Flint, J. Mapping in structured populations by resample model averaging. Genetics 182, 1263-1277 (2009).

45. Miao, C., Yang, J. \& Schnable, J. C. Optimising the identification of causal variants across varying genetic architectures in crops. Plant biotechnology journal 17, 893-905 (2019).

46. Adams, J., Qiu, Y., Xu, Y. \& Schnable, J. C. Plant segmentation by supervised machine learning methods. The Plant Phenome J. 3, e20001 (2020).

47. Kuhn, H. W. The hungarian method for the assignment problem. Nav. research logistics quarterly 2, 83-97 (1955).

48. Edmonds, J. \& Karp, R. M. Theoretical improvements in algorithmic efficiency for network flow problems. J. ACM (JACM) 19, 248-264 (1972). 


\section{Supplementary materials}

(A)

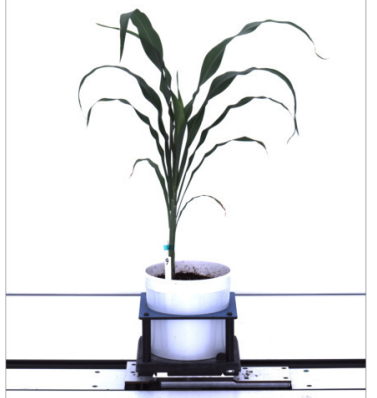

(B)

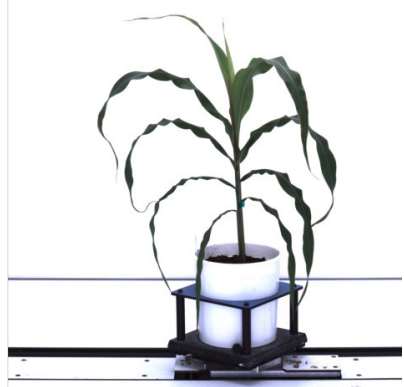

Figure S1. Two gentoypes having different extremes of leaf angles were selected to compare manual and automatic measurements of same plants.. (A) BTx623 (B) AS 4601 Pawaga.

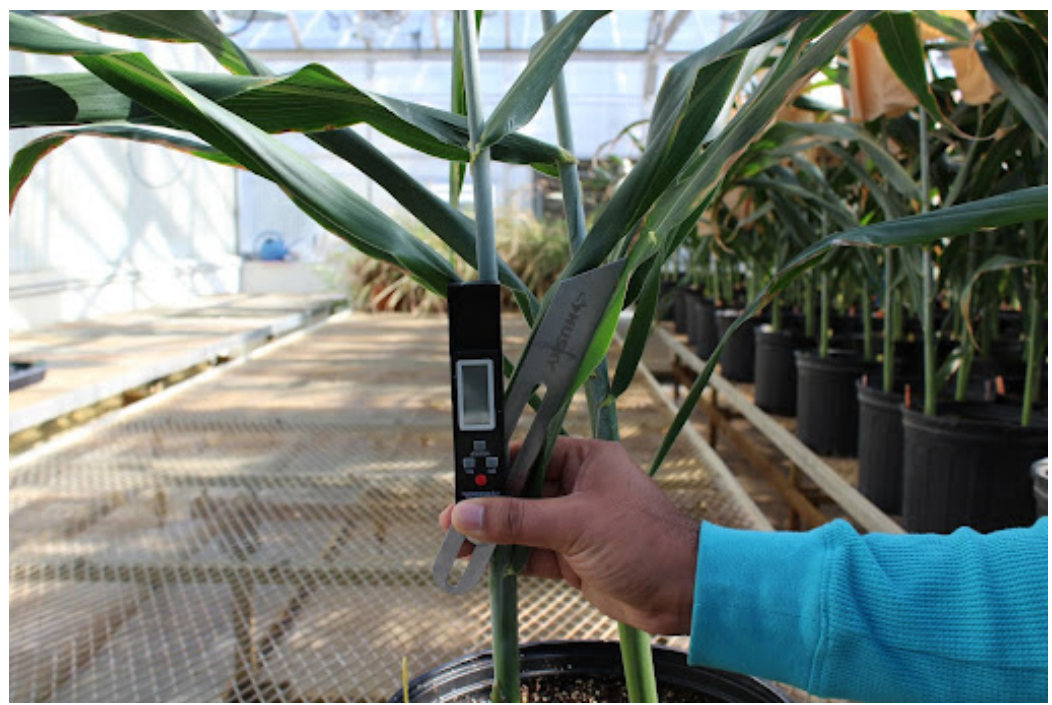

Figure S2. Example of manual leaf angle measurement in sorghum. An electronic protractor (Husky Sliding Digital T-Bevel/Angle Finder) is used to measure the interior angle between the sorghum stem and the midrib of the target sorghum leaf. 
(A)

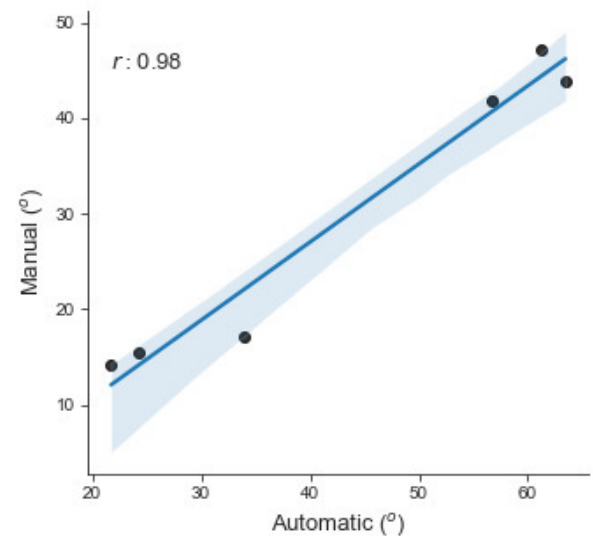

(B )

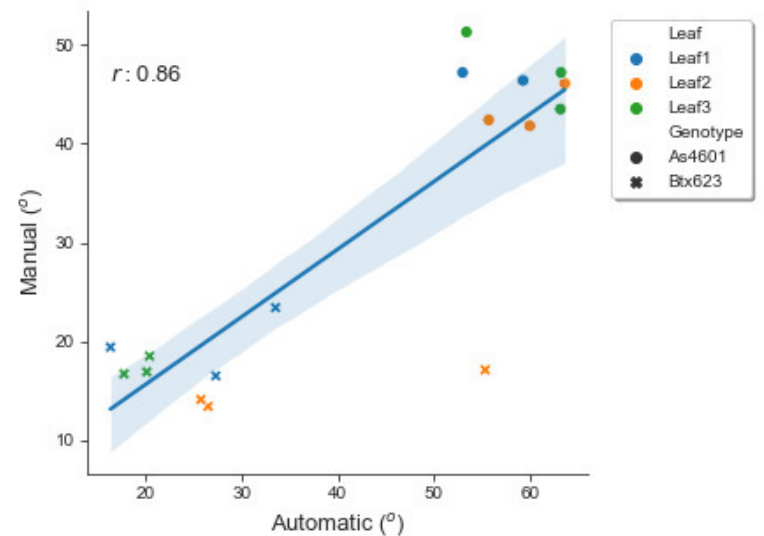

Figure S3. Automatically derived leaf angle measurements from 3D reconstructions are positively correlated with manual measurements of same plants. (A) Pearson $r$ correlations between the median leaf angle of leaves 1 to 4 derived from manual measurements of six sorghum plants representing phenotypic extremes for leaf angle in sorghum versus the median leaf angle of the same plants derived from automatic measurements of the 3D reconstructed plants. (B) Pearson $r$ correlations between manual measurements of the individual leaves 1 to 3 of six sorghum plants representing phenotypic extremes of leaf angle in sorghum versus automatically measurements of the same plants derived from 3D reconstructions.

(A)

(B)

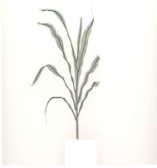

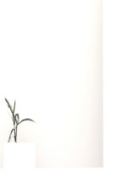

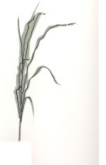

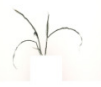

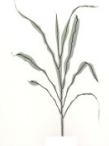

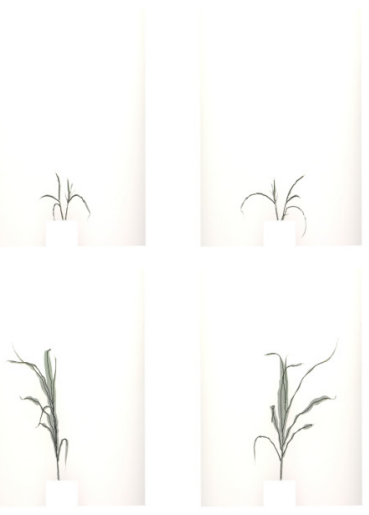

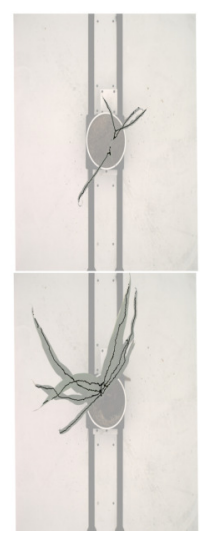

Figure S4. Overlay of outlier plants removed from all analyses and their optimized skeletons. (A) Leaf angles were large due to biological errors as a result of extremely poor growth and health under greenhouse conditions. (B) Leaf angles for plant was inconsistent with visual validation. 


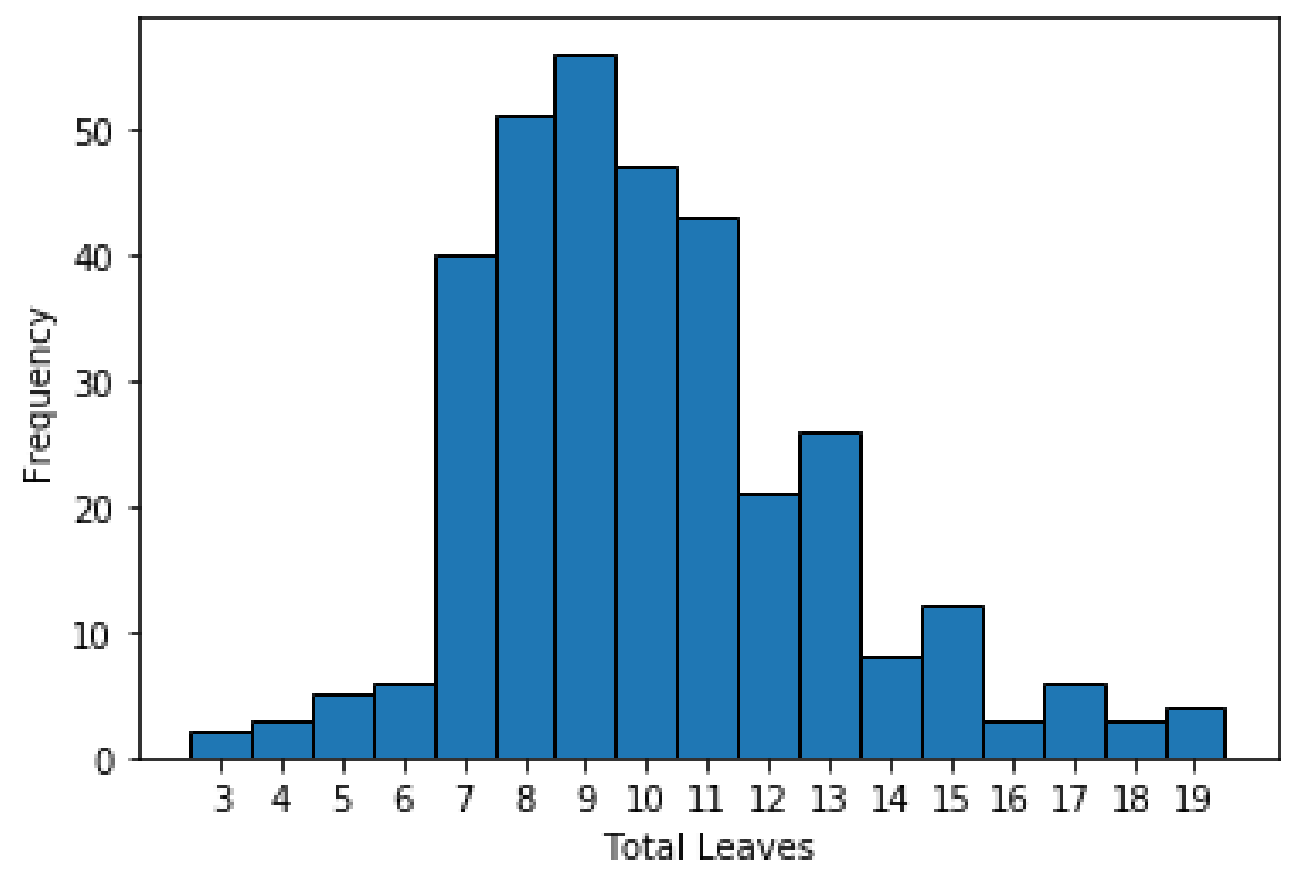

Figure S5. Frequency of the total amount of leaves of each plant

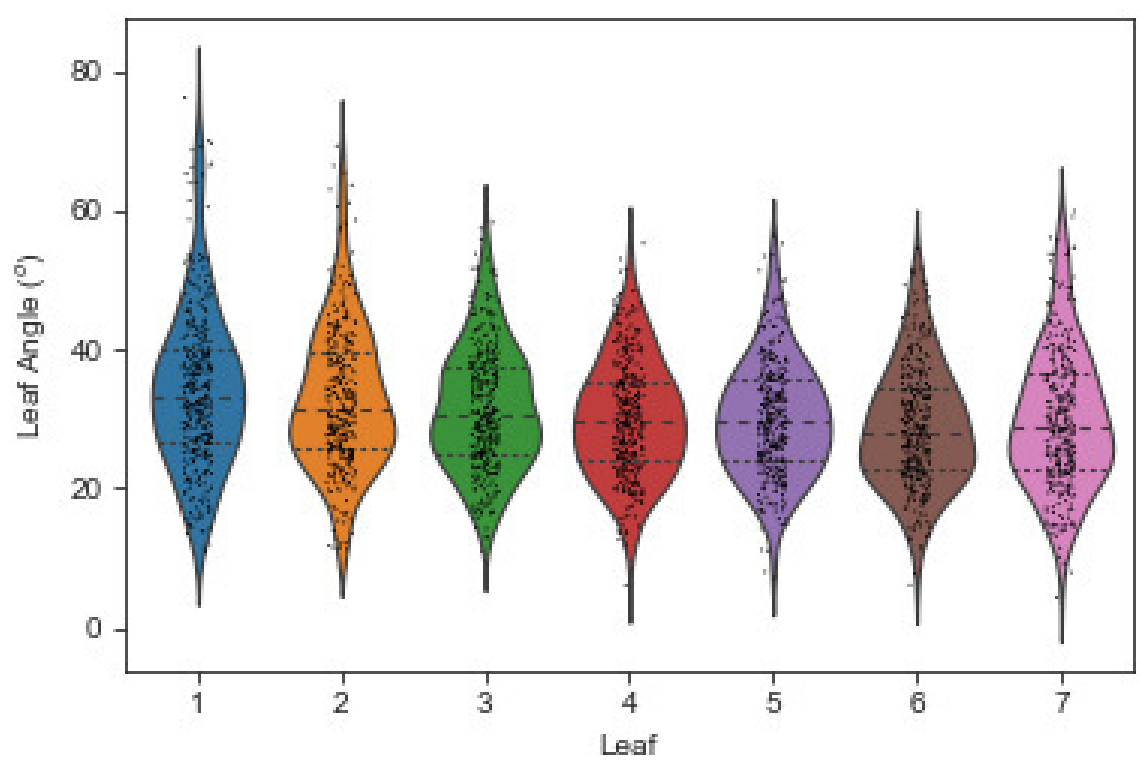

Figure S6. Violin plots illustrating the range of leaf angle values for each leaf 


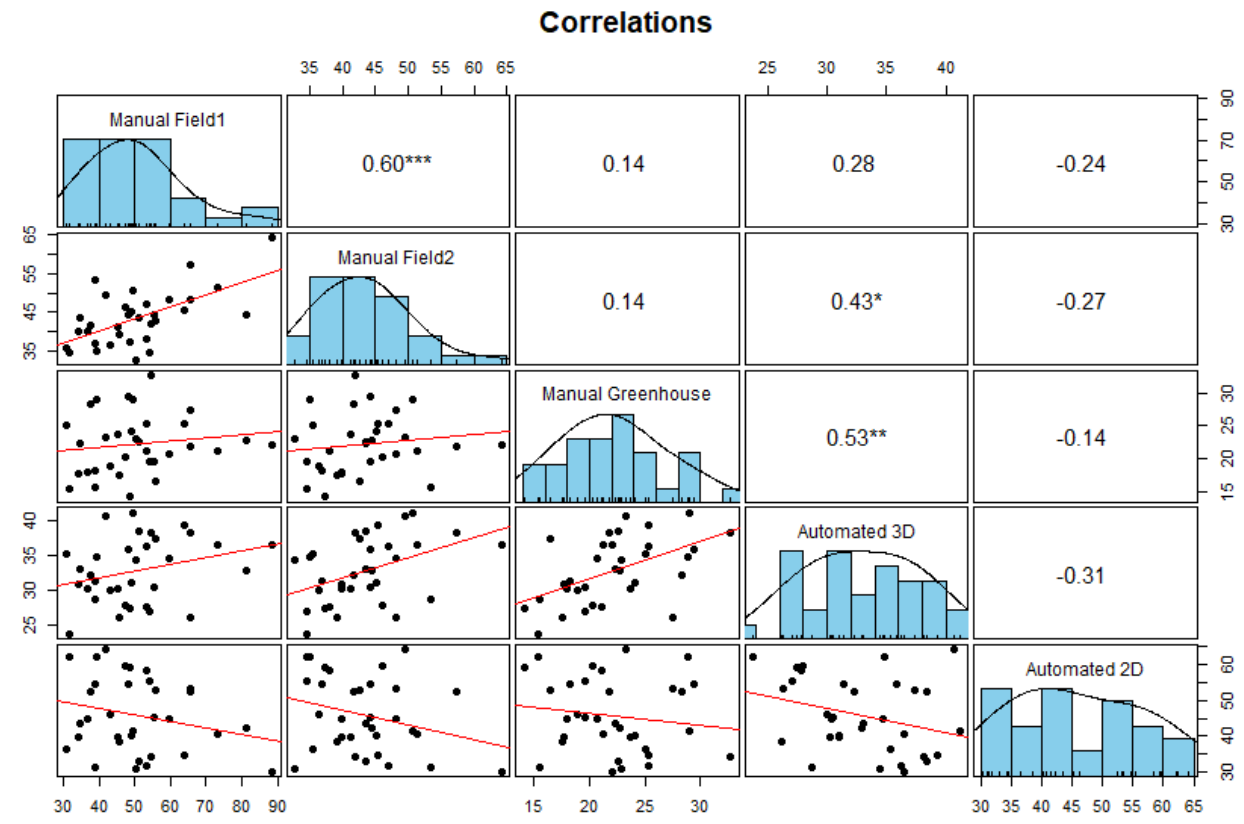

Figure S7. Pearson correlations and histograms of pairwise combinations of leaf angle datasets $(* * *=\mathrm{p}<0.001, * *=\mathrm{p}<$ $0.01, *=\mathrm{p}<0.05)$. Manual Field 1, Manual Field 2, Automated 3D and Automated 2D refers to the Iowa 2012 manual field data, the Nebraska 2020 manual field data, the Nebraska 2021 manual green house data, the Nebraska 2018 green house automatically derived 3D data and the Nebraska 2018 green house automatically derived 2D data. Stronger correlations were observed between datasets grown under the same condition and maturity stage (Manual Field1 vs Manual Field2 and Manual greenhouse vs Automated 3D ). Negative and non significant correlations observed between comparisons of automatically derived 2D measurements and all other datasets. 

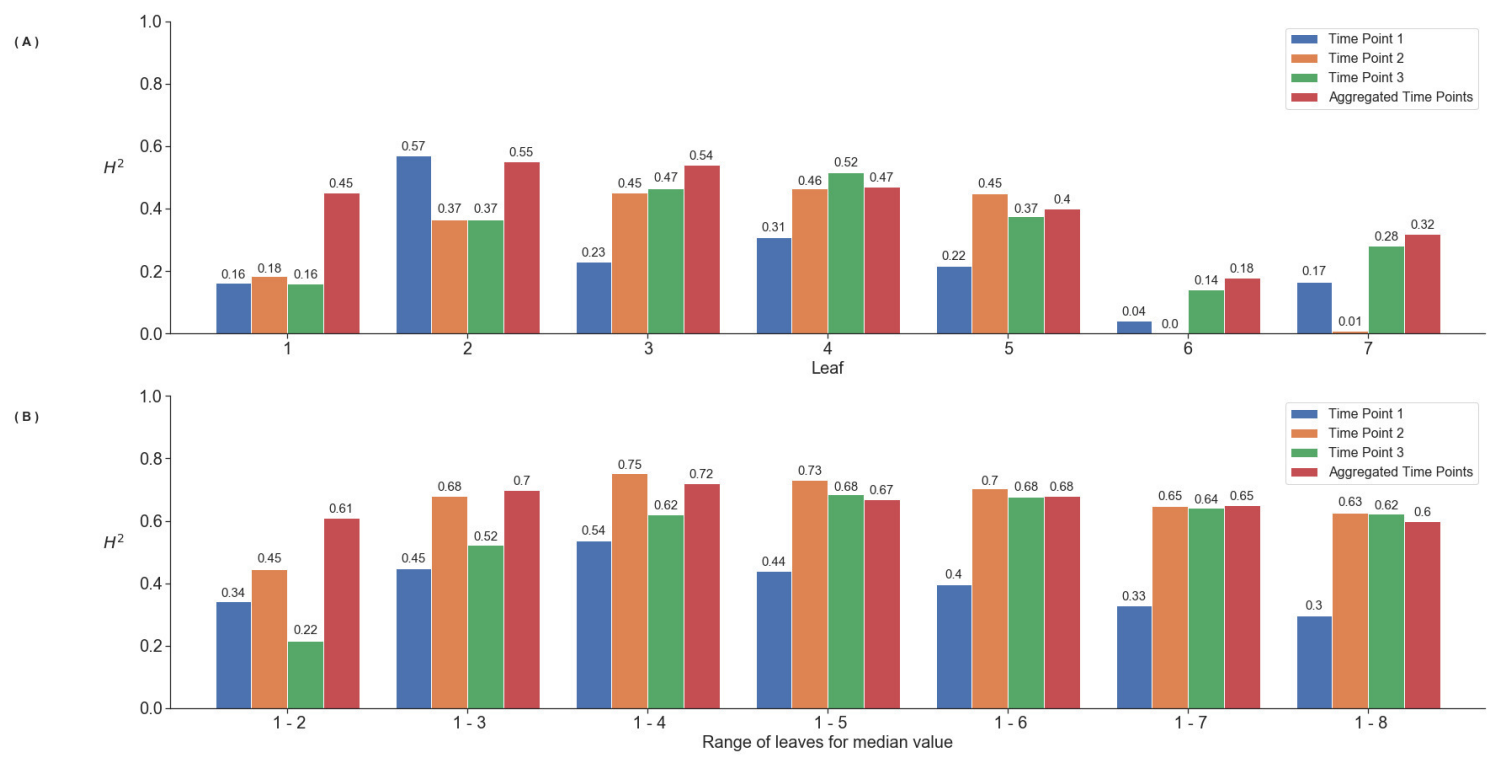

Figure S8. Heritability increases with the aggregation across time points and leaves within a plant (A) Heritability calculated for each individual leaf for each of the three time points as well as after aggregation of time points. (B) Heritability calculated using the median value of different leaf combinations for three different time points and after aggregation (The median angle of the three values of a single leaf, was assigned for that specific leaf and plant)

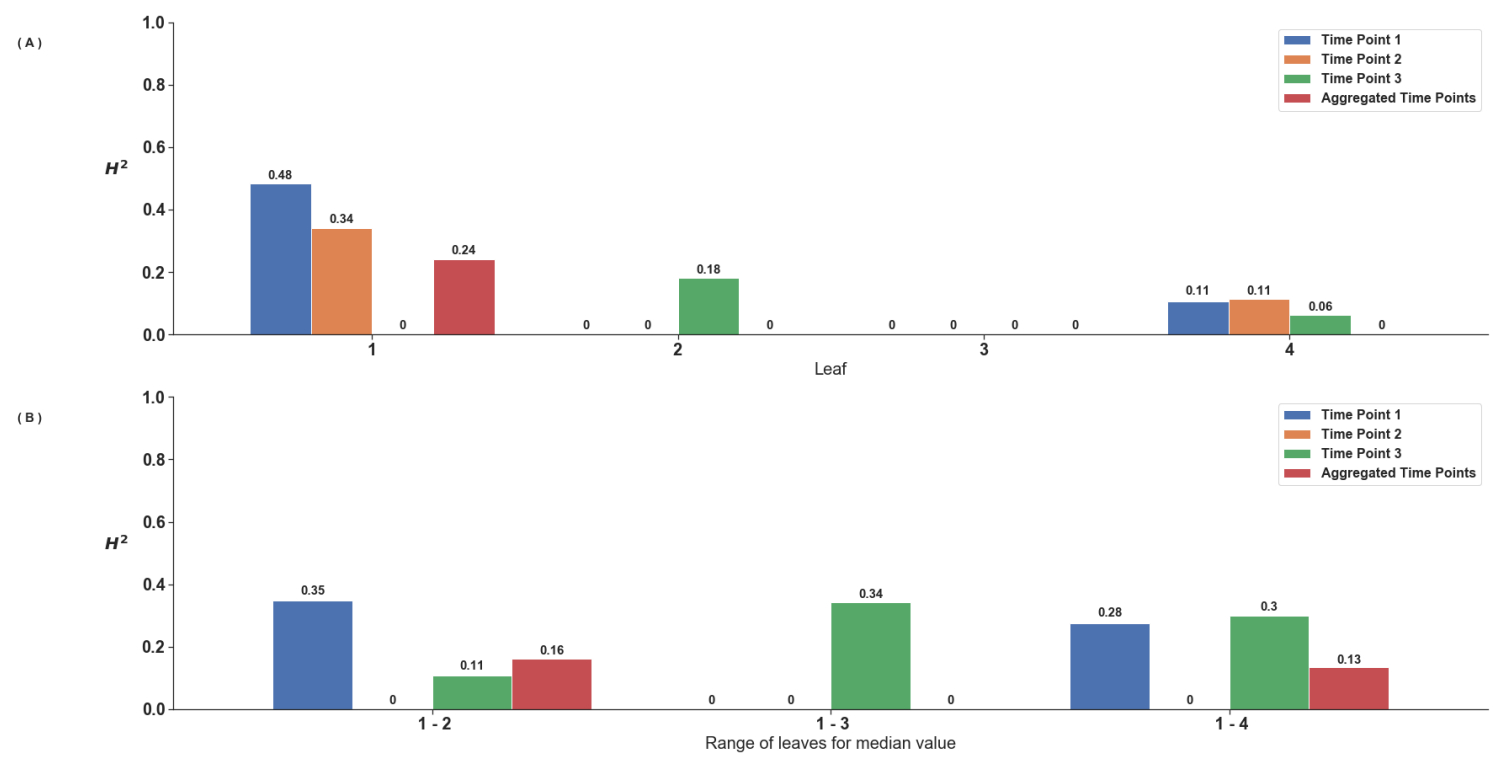

Figure S9. Lower heritability values generally observed in leaf angles obtained from automated 2D measurements (A) Heritability calculated for each individual leaf for each of the three time points as well as after aggregation of time points. (B) Heritability calculated using the median value of different leaf combinations for three different time points and after aggregation (The median angle of the three values of a single leaf, was assigned for that specific leaf and plant) 


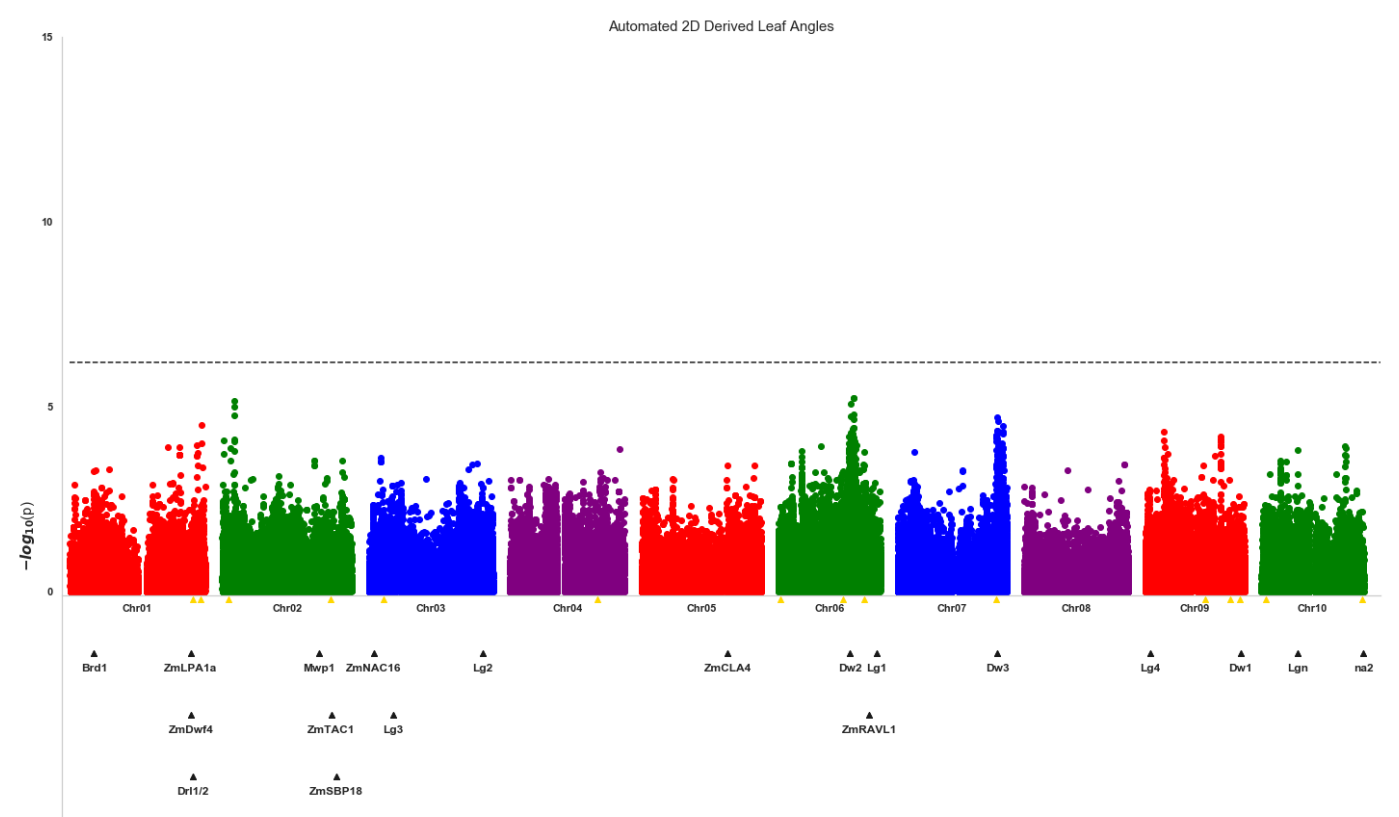

Figure S10. No genetic markers significantly associated with variation in sorghum leaf angle aggregated across leaves and time points with automatic 2D derived measurements . A Mixed linear model based approach implemented in GEMMA [39] was used to identify significant associations of SNP markers with variation in leaf angle aggregated across all three time points. Each point indicates the physical position and statistical significance of an individual marker. Dashed black lines indicate a genome wide threshold for statistical significance of a $6.39 \times 10^{-7}$ resulting from a bonferroni correction using an effective SNP number of 78,251 (See Methods). Yellow triangles indicate the locations of significant QTL for leaf erectness detected in an analysis of a sorghum NAM population [25]. Black triangles indicate locations of a set of cloned sorghum genes or the locations of the syntenic ortholog in sorghum of maize genes which are known to influence variation in leaf angle in maize and are located near significantly trait asssociated SNPs in this study 


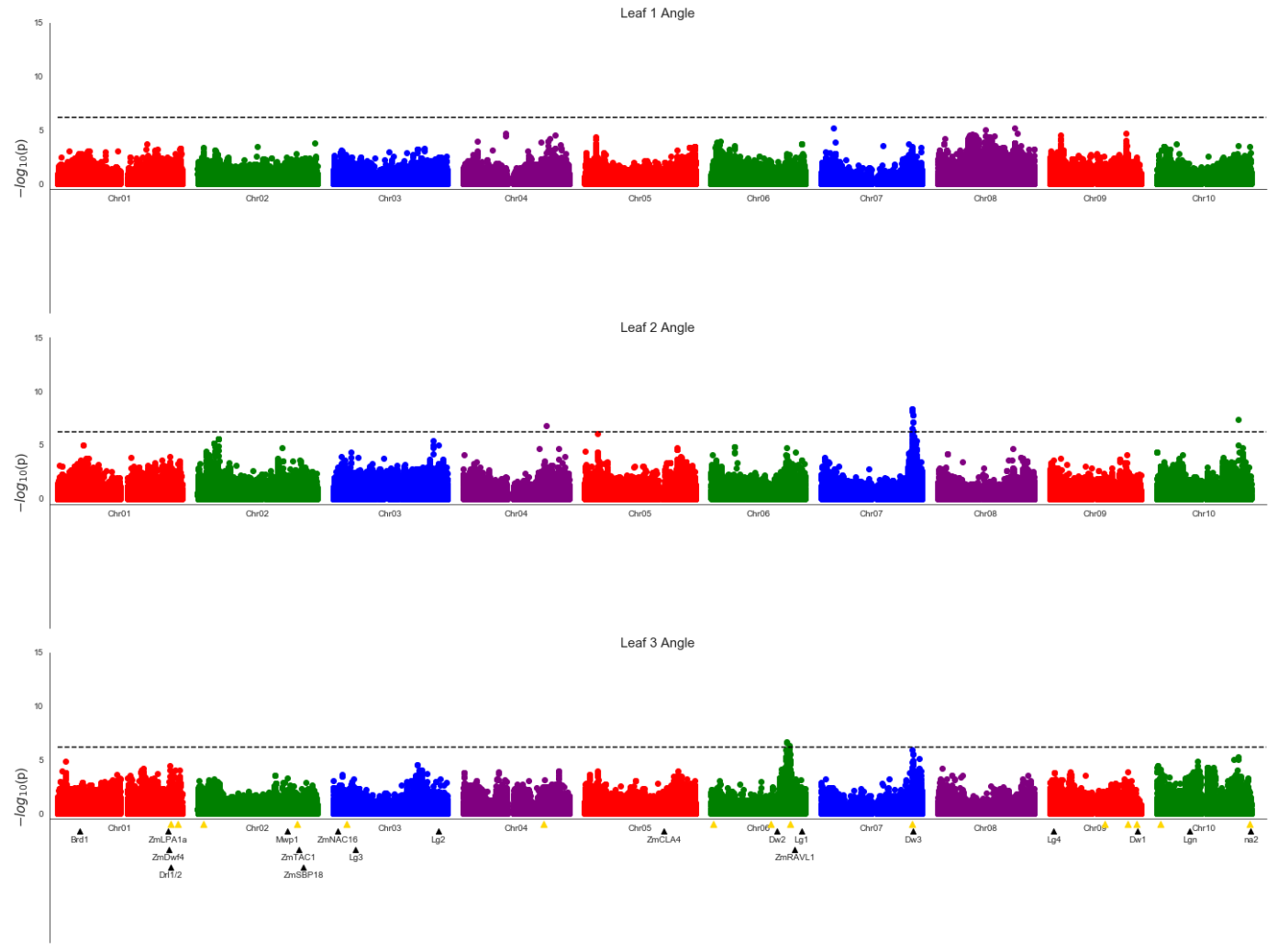

Figure S11. Genetic markers significantly associated with variation in the angle of leaves 1,2 and 3 . A Mixed linear model based approach implemented in GEMMA [39] was used to identify significant associations of SNP markers with variation in leaf angle aggregated across all three time points. Each point indicates the physical position and statistical significance of an individual marker. Dashed black lines indicate a genome wide threshold for statistical significance of a $6.39 \mathrm{x}$ $10^{-7}$ resulting from a bonferroni correction using an effective SNP number of 78,251 (See Methods). Yellow triangles indicate the locations of significant QTL for leaf erectness detected in an analysis of a sorghum NAM population [25]. Black triangles indicate locations of a set of cloned sorghum genes or the locations of the syntenic ortholog in sorghum of maize genes which are known to influence variation in leaf angle in maize and are located near significantly trait associated SNPs in this study 